\title{
Recent Insights into the Crystallization Process; Protein Crystal Nucleation and Growth Peculiarities; Processes in the Presence of Electric Fields
}

\author{
Christo N. Nanev \\ Rostislaw Kaischew Institute of Physical Chemistry, Bulgarian Academy of Sciences, 1113 Sofia, Bulgaria; \\ nanev@ipc.bas.bg; Tel.: +359-2-8566458 \\ Academic Editor: Abel Moreno \\ Received: 22 September 2017; Accepted: 12 October 2017; Published: 15 October 2017
}

\begin{abstract}
Three-dimensional protein molecule structures are essential for acquiring a deeper insight of the human genome, and for developing novel protein-based pharmaceuticals. X-ray diffraction studies of such structures require well-diffracting protein crystals. A set of external physical factors may promote and direct protein crystallization so that crystals obtained are useful for X-ray studies. Application of electric fields aids control over protein crystal size and diffraction quality. Protein crystal nucleation and growth in the presence of electric fields are reviewed. A notion of mesoscopic level of impact on the protein crystallization exercised by an electric field is also considered.
\end{abstract}

Keywords: protein crystallization; classical and two-step nucleation mechanisms; impact of electric fields on the protein crystallization; external and internal electric fields; number density; size and quality of protein crystals

\section{Introduction}

Crystallization is a ubiquitous process occurring in nature, technology, and even in biology (e.g., bio-mineralization of bone, teeth, and shells). Crystals are present in both healthy (insulin) and ailing humans (formation of kidney and gall stones, uric acid crystals in gout, amyloid fibrils and insoluble plaques, the latter been considered the causative agents in in some neurodegenerative diseases, such as Alzheimer's disease and Parkinson's disease). Due to the vital importance of proteins for all living organisms and protein biochemical involvements [1], three-dimensional protein structures attract a continuously growing research interest. When it comes to understanding the mechanisms of life and human genome, as well as developing novel protein-based pharmaceuticals [2], these structures prove to be of essential importance.

The preferred techniques for determination of three-dimensional protein molecular structures involve X-ray (and neutron) diffraction. However, they require crystals that are large enough [3], and well-diffracting. The well-known difficulties encountered in attempting to grow crystals of newly expressed proteins have prompted exploration of many diverse crystallization approaches. Related to these approaches is also the study of external physical factors, such as magnetic and electric fields (EFs); proper crystallization conditions can be fine-tuned using variation of both direct current $(d c)$ and alternating current $(a c)$ EFs. Pioneered by Aubry's group [4,5] some 20 years ago, protein crystallization under EF attracts an increasing attention, becoming a mature scientific branch today. Major contributions in this research field have been made by the teams of Moreno [6-17], Koizumi [18-29], Veesler [30-33], etc. (the list is not exhaustive). Traditionally, most of the experimental studies were performed with hen-egg white lysozyme (HEWL). Three reviews [10,31,34], and a book chapter [17] have already been published. 
This paper focuses on the extensive research done on protein crystal nucleation and growth in EF [4-47]. In addition, the effect of EFs on other substances, e.g., the simplest possible amino acid glycine $[48,49]$ and sucrose crystallization $[50,51]$, is considered. For clarity, the paper also presents some basic crystallization process peculiarities.

Recent experimental and theoretical advancements supporting better protein crystallization understanding are discussed by Giege [52]. Some novel super-resolution techniques enabling a profound insight into the mesoscopic and molecular level scenarios of protein crystal nucleation and growth are elaborated as well [53]; such techniques are compared below. The aim of this comparison is to provide clues to which one of them may be applied for studying mesoscopic level processes of protein crystallization in EFs.

Currently, Atomic Force Microscopy (AFM), Laser Confocal Microscopy enhanced by Differential Interference Contrast (LCM-DIM) technique and Michelson Interferometry (MI) are used to visualize molecular-level surface processes and crystal morphology, as well as to measure crystal growth rates. In contrast to AFM, LCM-DIM is a non-invasive optical method. With several case studies, Sleutel et al. [54] have demonstrated the strengths, limitations and weaknesses of these three techniques. AFM, LCM-DIM and MI have different vertical, lateral and time resolutions, and their complementary application is considered to be highly advisable. AFM at molecular-level resolution is the most suitable technique for observing slow nanoscale growth processes. LCM-DIM is a microscale observation technique with nanoscale vertical resolution, useful for slow to medium growth processes, while MI is best suited for fast growth processes requiring only micrometric lateral resolution. The data acquired by means of these techniques are very useful for developing reliable physical models relevant to the crystal growth issues at hand [55].

Finally, the recently developed time resolved liquid-cell transmission electron microscopy (LC-TEM) proves to be a very powerful tool to study protein crystal nucleation and growth. For instance, Yamazaki et al. [56] have used LC-TEM to understand the mechanisms underlying the early stages of protein crystallization (see Section 2.1).

\section{Crystal Nucleation}

Spontaneous crystallization, which is a common practice for protein crystal growth, starts with formation of the smallest possible stable crystalline particles under the actual conditions, coined nuclei. To evoke nucleation, the equilibrated system needs to be supersaturated, i.e., it is necessary to change system energetic status [57]. For instance, the solution is cooled to evoke crystallization (with normal solubility temperature dependence) and vice versa, it is heated to crystallize (with retrograde solubility); water is heated to boil, it is cooled to freeze, etc. The measure for the degree of energy-change is the imposed supersaturation, $\Delta \mu=\mathrm{k}_{\mathrm{B}} \cdot \mathrm{T} \cdot \ln \left(c / c_{\mathrm{e}}\right)$, which is the driving energy for a new-phase nucleation and further growth (where $\mathrm{k}_{\mathrm{B}}$ is the Boltzmann constant, $\mathrm{T}$ is the absolute temperature; and for solution, $c$ is the actual concentration and $c_{\mathrm{e}}$ is the equilibrium one).

Being the first crystallization stage, the nucleation process predetermines important features of the subsequent crystal growth, such as polymorph selection (which is an issue of great interest for the pharmaceutical industry, because the same molecule may or may not have a therapeutic effect depending on the crystal polymorph), number of nucleated crystals, crystal quality, and crystal size distribution. Although benefiting from 120 years of research on small molecule crystal nucleation, the process with proteins is far from being thoroughly understood. Governed by some physical laws found initially for the small molecule crystallization, protein crystal nucleation is extremely complex. This complexity arises from the subtle interplay between physics and biochemical idiosyncratic features of proteins. It is the large size of their molecules and their highly inhomogeneous, patchy surface (that is essential for protein biological role) that evoke specificity to the molecular-kinetic protein crystal nucleation mechanism [58]. 
Despite nucleation importance, direct observation of critical nuclei has proven elusive, even nowadays. The reason is the inherent impossibility to observe the molecular scale acts of crystal nucleation. Protein crystal nuclei make no exception. Although formed by huge protein molecules, being still nanosized particles, they remain invisible by optical microscopy. AFM is able merely to visualize elementary acts during protein and virus crystal growth [59,60], while the sizes of the protein crystal nuclei are determined by means of thermodynamic estimations. Using LCM-DIM, Sazaki's group studies the 2-D nucleation kinetics of lysozyme [61] and glucose isomerase crystals (under high pressure) [62]. However, the main difficulty in the experimental study of the crystal nucleation arises out of the fact that it is impossible to distinguish the critical nuclei in the whole assembly of under-critical, critical and super-critical molecular clusters; cluster composition changes dynamically due to the constant growth/decay of differently sized clusters; and critical nuclei are not labeled. That is why they are indistinguishable under mere observation (only growth of 2D clusters is visualized by the most powerful observation methods, such as AFM, LCM-DIM and LC-TEM).

\subsection{Evoking Nucleation; Classical Nucleation Theory (CNT) vs. Multi-Step Nucleation Mechanism}

All nucleation phenomena, whether they proceed homogeneously or involve foreign particles, surfaces, EFs, etc., require formation of an interface between the old (mother) phase and the newborn condensed phase. Gibbs [63] has pointed out the major thermodynamic aspect of the nucleation process, namely, the large barrier to phase transition associated with the energy cost for creating this interface. The classical nucleation theory (CNT) is fundamentally based on interphase fluctuations, needed to surmount this barrier.

Although CNT has provided a reasonable explanation of the fluctuation-based nucleation mechanism and the nucleation barrier origin, in some cases it has failed to predict correctly nucleation rates, with deviations being of many orders of magnitude. Debating this lack of adequacy, researchers have proposed multistep nucleation mechanisms, formulated initially as a two-step nucleation mechanism (TSN). Unlike most small molecules, proteins can take diverse aggregation pathways that make the outcome of crystallization assays quite unpredictable. Ten Wolde and Frenkel [64] have predicted theoretically the existence of amorphous nuclei precursors. It was shown that the latter exist to a significant extent even in (under-)saturated solutions [65]. Whitelam [66] presents a molecular model designed to study crystallization in the presence and absence of amorphous intermediates. Based on computer simulations, he suggests tuning the relative strengths of the specific and nonspecific interactions, thus enabling the study of the relative efficiencies of various pathways leading towards the final crystalline state. Using dynamic light scattering and optical microscopy (for measuring apparent induction time for the occurrence of the first crystal), Ferreira et al. [67] have suggested a new version of the multistep nucleation mechanism where concurrent aggregation pathways competing with crystal nucleation are considered. As confirmed by dynamic light scattering analysis, the nucleation of lysozyme crystals is preceded by an initial step of protein oligomerization and by the progressive formation of metastable clusters. Unfortunately, however, dynamic light scattering is unable to discern structured (crystalline) from amorphous (or liquid) clusters.

Cluster formation pathways are largely discussed in the multistep nucleation theories, however, being the core of CNT, the fluctuation-based nucleation mechanism is not denied. While preserving CNT basic concept (a fluctuation-based nucleation mechanism), TSN denies only the simultaneous densification and ordering during a single nucleation event. According to the initial TSN formulation [68], mesoscopic droplets enriched in protein appear in the protein solution. Being only densified, this intermediate phase preserves some similarity to the mother phase. Then, due to the reduced surface tension, the phase-transition energy barrier is lowered bellow the one needed for direct transition mother-phase-to-crystal (occurring via the CNT mechanism). Thus, crystal nucleation is greatly facilitated in the intermediate dense liquid. The second step in TSN is the formation of crystal nuclei inside the highly-concentrated regions. Evidently, TSN resembles Ostwald's rule of stages, which stipulates that a 
thermodynamically less-stable phase appears first, and then a polymorphic transition toward a stable phase occurs.

The existence of amorphous nuclei precursors has been confirmed experimentally by Vivares et al. [69], Sauter et al. [70], and further by Schubert et al. [71]. Sleutel and Van Driessche [72] have observed a non-classical nucleation for the 3D liquid-to-crystal transition of glucose isomerase; local increase in density and crystallinity do not occur simultaneously, but rather sequentially. They have demonstrated that at high concentrations $(\sim 100 \mathrm{mg} / \mathrm{mL})$, glucose isomerase can form mesoscopic liquid-like aggregates (the molecules retain enough mobility), which are potential precursors of crystalline clusters. These aggregates are stable with respect to the parent liquid and metastable compared with the crystalline phase. In contrast, glucose isomerase 2D crystal nucleation proceeds classically [73]; and the authors have proven the existence of a critical crystal size. Sleutel et al. also observed that, in this case, the interior of all clusters is in the crystalline state and the cluster dynamics are determined by single molecular attachment and detachment events [73].

According to most recent observations, however, the initial formulation of the TSN needs some redaction. This has been concluded by Yamazaki et al. [56] (who conducted experiments with LC-TEM). The authors have established that mesoscopic clusters, similar to those previously assumed to consist of a dense liquid and serve as nucleation precursors, are not liquid but amorphous solid particles consisting of lysozyme molecules. Moreover, lysozyme crystals never form inside them. Instead, nucleation events of orthorhombic lysozyme crystals attached to a silicon nitride window or to an amorphous solid particle are observed frequently. Nucleation is initiated with spherical particles which transform into faceted orthorhombic crystals. Under the tested experimental conditions, simultaneous formation of two lysozyme crystal polymorphs is observed, i.e., thermodynamically more-stable orthorhombic crystals and less-stable tetragonal crystals; the former grew further, while the latter dissolved. Moreover, orthorhombic crystals are more stable than amorphous solid particles under the experimental conditions. These observations clearly indicate that the amorphous solid particles act merely as a heterogeneous substrate that enhances the nucleation event, proceeding according to CNT. (In other words, the assumption that protein crystal nucleates heterogeneously on foreign particles of biological origin [74] is confirmed by these experiments). All this marks a significant departure from the initial formulation of the TSN [75].

Finally, an assessment of the balance between entropy and enthalpy for solute association to crystals is required to consider process thermodynamics. When incorporated into the crystal lattice, molecules lose the possibility to move freely in the solution. This results in entropy loss (that is due to the constrained translational and rotational degrees of freedom of the molecules), and disfavors crystallization. On the contrary, the release of some water molecules, attached to the contacting patches when crystalline bonds are formed, boosts system's entropy. Trapping and rearrangement of water also affect crystallization thermodynamics.

The entropic restriction is more important for protein crystallization due to the large size and complex shape of these molecules. Using LCM-DIM, Sleutel et al. [54] have determined the precise temperature dependent solubility of tetragonal lysozyme and glucose isomerase crystals. On this basis, the authors have characterized the thermodynamics of crystallization. Applying van't Hoff equation, they have calculated the standard free energy, enthalpy and entropy of protein crystallization. The conclusions are that the entropic effect is compensated by the larger enthalpy change, and that the crystallization process is exothermic [54].

Comparing enthalpic and entropic contributions to the free energy of pre-nucleation cluster formation in the $\mathrm{CaCO}_{3}$ system, Kellermeier et al. [76] (Table 1 in [76]) have noticed the minor costs in enthalpy linked to cluster formation, and conclude that the pre-nucleation cluster formation is predominantly driven by entropy. The entropic driving force is associated with the return of water to bulk solution; a gain in translational and rotational degrees of freedom is arising after water release from hydrated disordered precursors and water molecules move back into the surrounding solution. This drives the assembly of remaining solute molecules into an ordered (i.e., crystalline) structure. 
Moreover, the key role of water release suggests that pre-nucleation cluster formation may be a common phenomenon in aqueous solutions.

\section{Crystal Growth}

After crystals nucleate, they start growing immediately. In this second crystallization stage, the crystals grow until solution depletion reaches a level which corresponds to zero supersaturation with respect to the smallest crystal in the system; this point marks the beginning of the so-called Ostwald ripening [57].

Multistep crystal nucleation pathways involving liquid-like, amorphous or metastable crystal precursors challenge our current understanding of crystallization by putting the question: Can also some metastable crystalline precursors play a role during the crystal growth? Being predicted by theoretical works and observed experimentally at nucleation, there is only some evidence that metastable crystalline precursors can also be relevant to the growth of the crystals. With proteins Sleutel and Van Driessche [72] have shown a surface cleansing, triggered by mesoscopic clusters of protein molecules formed in bulk solution. Sedimenting on the crystal surface, and merging with it, the clusters form expanding mounds containing a considerable number (ranging from 2 to $>100$ ) of monomolecular steps. The expanding mounds trigger a step cascade that causes the self-purifying effect. If the impurity content of the arriving clusters is lower than the impurity concentration in the mother liquor, the steps propagating on the crystal surface, lead to its cleansing. The latter is a result of acceleration in the step velocity (which is due to the lower impurity concentration), and thus reduction of terrace exposure time with respect to impinging impurity.

Quite recently, Jiang et al. [77] have shown that disordered nanoscopic precursors can also play an active role in the stage of growth of organic compound crystals. Using in situ AFM on the $\{110\}$ facets of a preexisting crystalline $\mathrm{Glu}_{\mathrm{H}} \mathrm{H}_{2} \mathrm{O}$ surface, the authors have observed that prenucleation clusters are involved during growth of DL-glutamic acid crystals. This non-classical scenario of growth proceeds through attachment and transformation of 3D nanoscopic precursors units (larger than the monomeric constituents) which finally transform into crystalline 2D nuclei; the latter eventually build new molecular layers by further monomer incorporation. Moreover, under a direct observation, the 3D nanospecies act as an initial material depot for subsequent epitaxial growth. The preexisting crystalline surface plays a crucial role in decreasing the barrier to epitaxial growth via heterogeneous nucleation. (On the opposite, due to lattice mismatch, the silicon surface lacks sufficient structural similarity to promote formation of 2D nuclei.) These results have been confirmed using three independent methods, such as electrospray ionization mass spectrometry, analytical ultracentrifugation and in situ AFM on an inert silicon substrate.

Sleutel et al. [78] have found that incorporation of growth units to crystal surface steps occurs through surface diffusion. As opposed to direct incorporation from solution where all these events need to operate in a concerted way and (therefore) result in a large activation barrier, surface diffusion is a two-step process where the barriers for adsorption and incorporation into the step are separated. Unexpectedly, proteins can also grow by the 2D nucleation mechanism even at low supersaturation due to the lack of active spirals on the crystal face [54]. Transition from 2D-nucleation to kinetic roughening of glucose isomerase crystals with supersaturation increase has been observed directly using LCM-DIM [79]. In such studies, computer simulations [80] can be very useful for predicting crystal growth.

\subsection{Crystallization in the Presence of Electric Fields (EFs)}

\subsubsection{EFs Affect Protein Crystal Number Density and Improve the Quality of the Crystals Grown}

Initially, EFs have been applied externally (e.g. [4,5,39]); that is to say, no contact between the electrodes and the solution. Systematic studies on the effect of a static EF have been carried out first by the Aubry's group [4]. The authors have also focused on providing a theoretical explanation of EF 
distribution (potential difference) inside the crystallizing system. Due to the high solution conductivity, the electrostatic potential change penetrates only in a thin solution layer but not in the solution bulk. With the aid of a vapor diffusion method, the authors show that EF suppresses HEWL crystal nucleation while simultaneously improving the diffraction quality of crystals grown (as estimated from the rocking-curve measurements conducted). It is assumed that EF directs HEWL molecules to fall oriented on the crystal surface, thus contributing to the improved crystal quality. The authors have also observed growth of HEWL crystals at the droplet surface near the cathode. Keeping in mind that, under the experimental conditions set, HEWL molecules are positively charged, this result follows the basic law in electrochemistry. When the voltage is higher than $1000 \mathrm{~V}$, the drop starts to move towards the cathode. Subsequently, Aubry and coworkers [5] have measured directly protein concentration changes appearing upon application of an external EF and lead to a concentration gradient between the electrodes, the highest HEWL-concentration being observed near the cathode. This increased local supersaturation explains why EF affects protein crystallization.

Using custom-made 2D glass cells to crystallize HEWL, and simultaneously control temperature and substantially reduce convection, Nanev and Penkova [39] have applied an external high voltage $(1500 \mathrm{~V} / \mathrm{cm})$ static EF. To ensure uniform EF in a flat condenser, two silver plates charged negatively and positively are pressed to the upper and bottom glass cell windows; in different experiments, the cathode is placed on the bottom or on the top of the cell. Under such conditions, EF is at its maximum in the solution adhering to the glass surface and decays rapidly towards solution bulk. It has been confirmed that HEWL crystals grow predominantly on the cathode side of the glass cell. In EF these crystals grow to visible sizes in less than $2 \mathrm{~h}$, most of them being oriented with their c-axis normal to the supporting glass plate. Figure 1 shows highly predominant c-axis orientation of HEWL crystals (despite EF uniformity, deviations in the percentage of c-axis oriented crystals are noted at different places of the glass support). However, this preferred crystal orientation is reported to occur only at temperatures below $5-7^{\circ} \mathrm{C}$, down to $0{ }^{\circ} \mathrm{C}$, while missing at higher temperatures, $18{ }^{\circ} \mathrm{C}$ and above.

It is logical to assume that $\mathrm{EF}$ orients HEWL molecules during the stage of protein crystal nucleation, and thus, predestinates crystal orientation. Applied through the (insulating) glass plate, only a small fraction of the external high $d c$-voltage affects HEWL crystallization. Therefore, its effect is observed merely at low temperatures, while, at higher temperatures, it is the thermal motion that prevails and disturbs protein molecule ordering, and prevents acquiring the preferred c-axis crystal orientation.

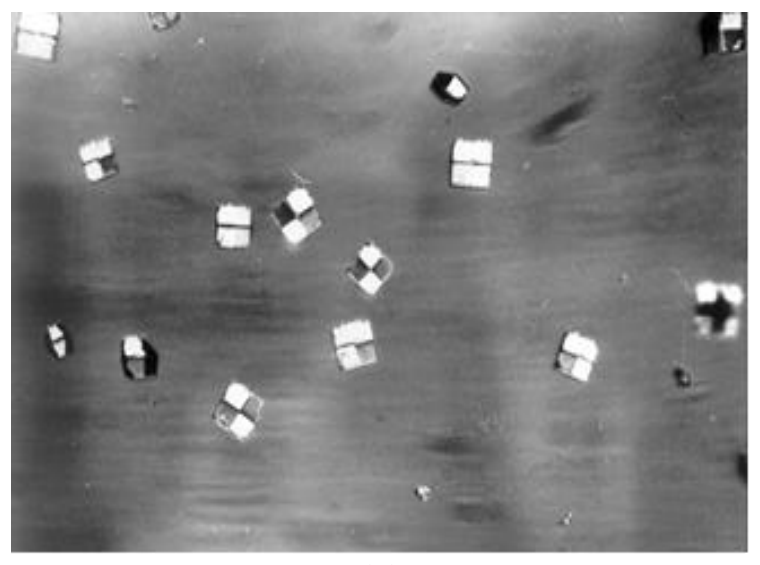

(a)

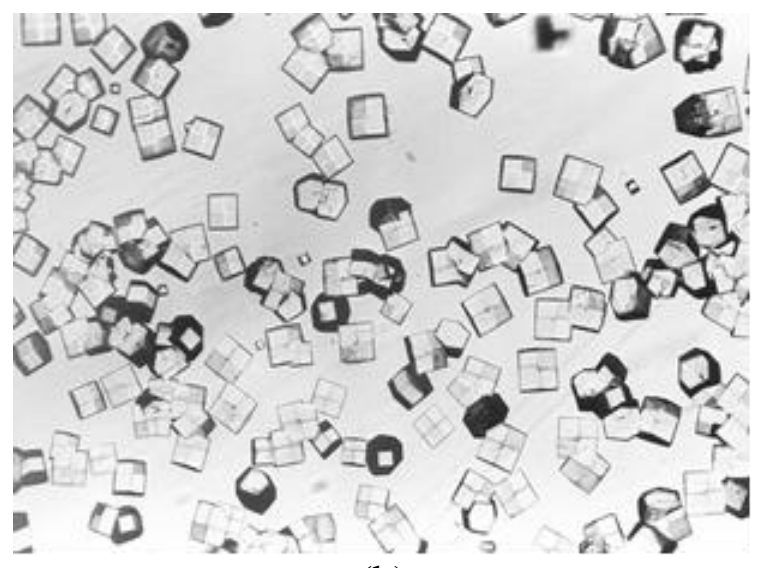

(b)

Figure 1. Preferred orientation of HEWL crystals on the cathode side. Imposed EF $=1500 \mathrm{~V} / \mathrm{cm}$, $20 \mathrm{mg} / \mathrm{ml} \mathrm{HEWL}$ and $0.7 \mathrm{M} \mathrm{NaCl}$. Crystal sizes: $35 \mu \mathrm{m}-70 \mu \mathrm{m}$. (a) Dark field image, $t=0{ }^{\circ} \mathrm{C}$. (b) Bright field image, $t=5{ }^{\circ} \mathrm{C}[39]$ (with permission from [39]). 
In addition to HEWL, Penkova et al. [40] have performed experiments on EF-assisted crystallization of ferritin and apoferritin in a sitting drop setup. The uncovered air/solution interface introduces complexity to the phenomenon; depending on the field strength, there occurs a solution stirring at rates of up to $100 \mu \mathrm{m} \mathrm{s}^{-1}$. At slow solution flow rates, nucleation of ferritin and apoferritin crystals is suppressed, while faster stirring enhanced crystal nucleation of both proteins.

As already mentioned, high-quality and relatively large protein crystals are needed for protein structural crystallography based on X-ray (and neutron) diffraction. The excellent potential of external $d c$-EFs for growing such crystals has been confirmed most recently [41]. Via X-ray diffraction analysis it is proven that glucose isomerase crystals grown (by the microbatch method at room temperature) in the presence of $d c$-EFs of 1,2, 4, and $6 \mathrm{kV}$ are of higher quality as compared with crystals grown in the absence of EFs. Light microscopy observations indicate a decrease in crystal nucleation rate and an increase in crystal size with the increase in voltage applied. This could be seen in Figure 2 (Figure 7 of Rubin et al. [41]).

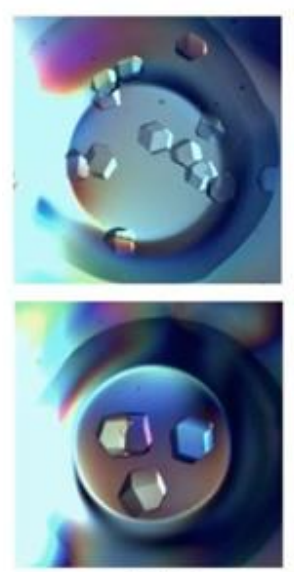

$0 \mathrm{kV}$

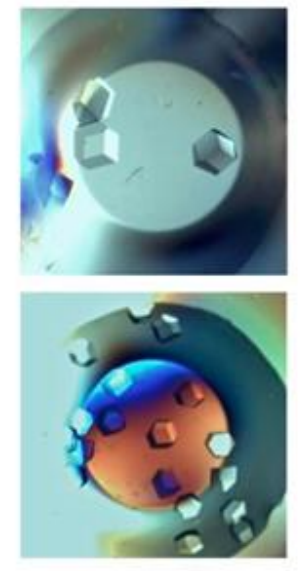

$1 \mathrm{kV}$

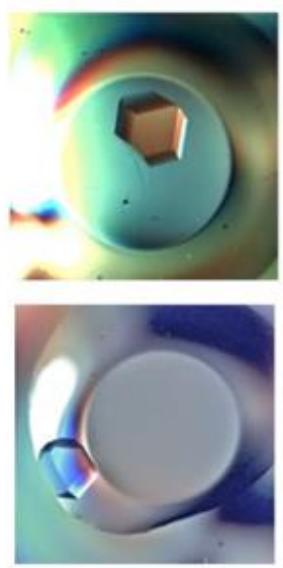

$6 \mathrm{kV}$

Figure 2. Glucose isomerase crystals grown in the presence of different $d c$-EFs intensities for 48 -h periods [41] (by permission of V. Stojanoff). The top and bottom panels are two typical experimental results.

An EF (electric potential difference) that injects a $d c$ between electrodes immersed in the solution (conventionally used in the following consideration as "internal EF"), has been first applied in the laboratory of Moreno [6]. The authors merge capillary tubes and gels for studying electrochemically-assisted crystallization of lysozyme and thaumatin. Applying X-ray diffraction structure analysis for assessing crystallographic data of the grown crystals, Mirkin et al. [6] have revealed the good potential of this protein crystallization type. In addition, using the gel acupuncture method, cytochrome $c$ crystallization has been expedited by a 15-day application of a constant current of $0.8 \mu \mathrm{A}$ [9]. A review of the significant progress in the electrochemically-assisted protein crystallization achieved until 2008 has been presented by Frontana-Uribe and Moreno [10] (see Sections 4 and 5). The authors also point out the significant difference between the electrochemically-assisted protein crystallization and the true electrochemical reaction accompanying it. During the former, there is no redox reaction occurring between the protein and the inert electrodes (e.g., Pt and graphite) immersed in the crystallizing protein solution, but only an EF-steering of the proteins, similar to electrophoresis. This results in protein molecules concentrating near the electrode. In contrast, during an electro-crystallization per se, the current flows through the electrolyte because EF is applied to the electrodes immersed in a solution containing small-molecule ions. As a result, cations (positively charged ions) migrate toward the cathode and anions (negatively charged ions) move toward the anode. On the electrodes, the solution ions give up their charges, and the substance making up the ions is liberated; e.g., in water solutions, hydrogen and oxygen gases are released, respectively, on the 
cathode and the anode. Despite solution electrolysis however, no bubbles appear provided the current densities are low enough (because the small gas amounts dissolve in the solution).

$d c$-electrochemically-assisted batch crystallization of lysozyme and ferritin has continued to be the focus of Moreno's interest while working (as a visiting professor) with Sazaki in his lab in Japan [7]. They have observed that applying a $d c$ of $2 \mu \mathrm{A}$ flowing between electrodes of platinum wires, the number of deposited lysozyme crystals significantly decreases, while the size and the quality of crystals increase [7]. Nucleation induction time for crystallization also decreases. Apart from platinum, graphite electrodes have been used later in electrochemical Hull type cells adapted for protein crystallization [12]. Orthogonal cells of this type produce the largest size and the highest quality of lysozyme crystals in solution as well as in gel.

A novel transparent crystallization cell, composed of two indium tin oxide (ITO) covered (conductive) glass plates serving as electrodes, have been employed by Gil-Alvaradejo et al. [11]. X-ray diffraction analysis indicates an improved quality of lysozyme crystals grown at $d c$ of $6 \mu \mathrm{A}$, and of ferritin crystals grown at $d c$ in range of 2 and $6 \mu \mathrm{A}$. No conformational changes in the 3D protein molecule structures are noted. The strong adhesion of protein crystals enables their characterization by in situ AFM. Such ITO-covered-glass-electrodes have been widely used in subsequent studies of Moreno's group. For instance, the electrodes have been adapted to a sitting-drop vapor-diffusion crystallization setup applied for lysozyme and 2TEL-Lys crystallization [13]. As observed by the authors, the lysozyme crystals growing while attached to the cathode are larger than those grown in the absence of an electric current.

Similar transparent ITO-covered glass cells have also been used by Wakamatsu and Ohnishi to study HEWL crystallization [35]. The authors employ various voltage waves, including sine, triangular and step waves at frequencies of up to $15 \mathrm{MHz}$, or dc. Wakamatsu [38] has tested ITO-based transparent cells for thaumatin crystallization in an extremely low internal ac-EF (a sine-wave voltage of $1.06 \mathrm{~V}$ at $20 \mathrm{~Hz}$ for $10 \mathrm{~h}$ ). By means of the same transparent crystallization cell, a lysozyme molecule aggregate formation has been studied by applying an internal EF and a low-angle $\left(<8^{\circ}\right)$ dynamic light scattering technique [36]. The method and the apparatus used for characterization of the protein aggregation have been described in detail elsewhere [37]. Differently ITO-patterned on-glass-slides-electrodes have served as bottoms of micro fluidic devices with parallel electrodes prepared for the study of protein (lysozyme and insulin) crystallization [42].

In general, an EF-introduced potential energy landscape can be shaped by adapting different types of electrodes. To apply an ac-current injection field during protein crystallization, Hou and Chang [47] have constructed interdigitated and quadrupole Ti/ Au electrodes to study the competition between gel and crystal formation at different voltages and frequencies. While applying EFs, there occurs a reduction in the nucleation site numbers, accompanied by a rapid increase in lysozyme crystal size. A method for protein crystallization involving the use of a microbatch under oil (where the crystallizing protein is contained in a small droplet of solution dispensed on electrically isolated electrodes) and a relatively low voltage (30-270 V) has been developed by Al-Haq et al. [46].

A crystallization cell with at least one sharp electrode has been proposed by Hammadi et al. [30]. To take control over the spatial and temporal location of the nucleation event, Hammadi et al. [32] have applied a localized (internal) $d c$-EF. The nanometer size of the electrode tip causes large EFs with steep field gradients and a high current density appearing in the solution close to the tip. Agarose gel is used to diminish convection. The experiments have been performed with bovine pancreatic trypsin inhibitor and lysozyme. To control the nucleation event location, the authors have employed the confinement effect of small droplets produced by microfluidics technologies coupled to external $\mathrm{EF}$ [33].

The combined effect of a $2 \mu \mathrm{A} d c$-EF and a $10 \mathrm{~T}$ homogeneous magnetic field has been studied as well [8]. A significant increase in both crystal size homogeneity and ratio of magnetically orientated crystals as compared with the controls is reported. Most recently, some sophisticated techniques for protein crystallization using $d c$ (in the range of 2-6 $\mu \mathrm{A}$ ) and $a c$ (in the range of 2-8 Hz) have 
enabled control over crystal nucleation, size and orientation (the latter governed by a rather strong magnetic field of $16.5 \mathrm{~T}$ ) [16]. These techniques allow for obtaining large and suitable crystals not only for high-resolution synchrotron X-ray, but also for neutron-diffraction crystallography. Combining adequately these modern experimental methods and involving nuclear magnetic resonance, the authors have determined the crystal lattice contacts for two model proteins, lysozyme and glucose isomerase. Another combination of $2 \mathrm{~Hz}$ ac-EF with a strong magnetic field of $16.5 \mathrm{~T}$, and radiofrequency pulses of $0.43 \mu \mathrm{s}$, have been applied to study the growth of lysozyme crystals in both solutions and gels [15]. It has been established that, using all types of precipitants (salts, organic solvents, polyethylene-glycols, etc.), these crystallization conditions render high-quality lysozyme crystals. Thus, the authors have grounds to suggest this crystallization approach as an alternative of the agarose gel crystallization (although the polyethylene glycols are popular precipitating agent for obtaining high-quality crystals, agarose does not polymerize in the presence of polyethylene glycols).

In a series of works, Koizumi et al. [18-29] have achieved a breakthrough in EF-promotion of protein crystal nucleation. Previous experience and theoretical considerations has given them reason to realize that the difference in electrical permittivity of liquid and solid must be the factor that defines the degree of change (appearing due to the external EF) in the chemical potential, $\Delta \mu$ (nucleation driving energy). The larger the difference in the electrical permittivity, the greater the effect of an externally applied EF on the nucleation rate. Since this electrical permittivity difference depends on $a c$-EFs frequency, HEWL crystal nucleation rate increases significantly when a high frequency $(1 \mathrm{MHz})$ external EF is applied, and decreases when an EF of $10 \mathrm{kHz}$ is applied. The nucleation rate in a $500 \mathrm{kHz}$ EF has been reported to be almost the same as the one in the absence of EF [18]. Thus, demonstrating both enhancement and retardation in the nucleation rate of HEWL crystals, the authors succeed in controlling effectively the process. Koizumi et al. [23] have also shown increase in crystal nucleation rate of porcine insulin by applying ac-EF of $3 \mathrm{MHz}$.

Furthermore, Koizumi et al. [19] have established that the electric double layer effect expressed at the solution drop/sealing oil interface is complementary to the effect of an external $a c$-EF. The authors have calculated that the external EF strength required to change the nucleation rate is $\sim 10^{4} \mathrm{~V} / \mathrm{cm}$, which is much larger than the experimentally applied EF of $800 \mathrm{~V} / \mathrm{cm}$. It turns out however that the important factor in changing the nucleation rate is the huge EF which is sustained in the electric double layer arising at the interface solution drop/oil; an electric potential difference of $\sim 1 \mathrm{mV}$ in a $\sim 1 \mathrm{~nm}$ thick electric double layer corresponds to an external EF of $\sim 10^{4} \mathrm{~V} / \mathrm{cm}$ (that is the calculated EF-strength). This finding has been experimentally confirmed in HEWL crystallization in an external $a c-\mathrm{EF}$ of $800 \mathrm{~V} / \mathrm{cm}$ at frequencies of 1-9 MHz. The authors have observed that nucleation occurs only on the surface of the solution drop but not in solution bulk.

The greater the ionic strength of the solution (dependent on the precipitant used), the thinner the electric double layer at the solution drop/sealing oil interface, and the stronger the EF-effect exerted on the supersaturation (being a crystal nucleation driving factor) [20]. The effect of an external $a c-E F$ on the HEWL crystal nucleation rate increases with the increase in the concentration of the precipitant used (in the case considered, the precipitant is $\mathrm{NiCl}_{2}$ ). The reason is that the electric double layer becomes thinner with the increase in precipitant ionic strength [21].

Of prime importance to the growth of well-diffracting crystal is to understand how imperfections originate in protein crystals. By means of X-ray diffraction rocking-curve measurements of HEWL crystals grown in the presence/absence of an external $1 \mathrm{MHz}$ ac-EF, Koizumi et al. [24,25] have shown that the quality of HEWL crystals improves when such $a c-E F$ is applied during crystal growth; the local strain in the crystals decreases significantly. This could be explained by the electrostatic energy of EF that is added not only to the chemical potential but also to the entropy [81] of the liquid and solid phases. Lowering entropy of the solid, the external $1 \mathrm{MHz} a c$-EF imposes higher structural order in the growing crystals. The authors believe that certain disorder in the protein crystals may be attributed to water molecule irregularities included in the crystals. When such irregularities are eliminated, the external $a c$-EF improves the homogeneity of tetragonal HEWL crystals. In contrast, when applying an 
external ac-EF of $20 \mathrm{kHz}$, Koizumi et al. [27] have observed a local strain accumulation in tetragonal HEWL crystals grown during nine days. The theoretical analysis suggests that this frequency has a significant effect on the liquid entropy. The rocking-curve measurements of HEWL crystals grown in the presence/absence of an external ac-EF, carried out by Koizumi et al. [26,28], have shown that no dislocations but rather misorientation among subgrains in the crystals are responsible for their inhomogeneity and strain. The authors show that such misorientations result from incorporation of impurities (most probably lysozyme dimers) into protein crystals. These impurities are integrated into the steps on the crystal surface during crystal growth, and also between adjacent subgrains. To elucidate the effect of $1 \mathrm{MHz}$ ac-EF on the elementary surface steps dynamics, Koizumi et al. [29] have measured (by means of microscopic observations) the growth rates of (110) and (101) faces of tetragonal HEWL crystals. The comparison of these data with the experimental results in the absence of EF shows a decrease in the growth rates of both faces under the applied $a c$-EF. This EF-effect is attributed to an increase in the effective surface energy of the step risers. The effects of a relatively low frequency ac-EF (less than $1 \mathrm{kHz}$ ) on HEWL crystal nucleation have been studied by Pan et al. [45], who conclude that such $a c$-EFs affect the mutual orientation between neighboring protein molecules.

\subsubsection{Potential of EF in the Selection of Protein Crystal Polymorph}

The dissolution rate of crystals and the delivery of drugs from crystal-containing formulations depend on the crystal polymorphic form. However, in a supersaturated solution, multiple polymorphs may appear. Hence, control over the polymorphic form is of importance for the pharmaceutical industry. Crystalline drugs must be manufactured in a specified polymorphic form only, and its purity is essential to its pharmaceutical application.

Evidently, the critical step in crystal polymorph selection lies in achieving control over nucleation stage. Having this in mind, Moreno's group [14] has chosen to apply a synergistic combination of complementary research approaches and tools (such as the use of temperature dependent solubility diagrams, X-ray diffraction and AFM, and molecular replacement method for structure determination) to study the temperature effect on glucose isomerase crystal polymorphism using a vapor diffusion setup with an ITO-electrodes crystallization cell. In doing so, the effect of $2 \mu \mathrm{A} d c$-EF on crystal nucleation and the optimal temperature for growing the best crystals has been explored. On the other hand, Kwokal and Roberts [44] have employed the electrochemical templating effect to look into the crystallization intricacies of different entacapone polymorphs using low magnitude $d c$-polarization of $\mathrm{Au}(100)$ and $\mathrm{Au}(111)$ interfaces.

Spherulitic crystals (crystals formed from thin needles growing radially outward from a center) may have a different morphology from the one of bulk crystals and, hence, dissolve differently. To study the number ratio between spherulitic and tetragonal HEWL crystals, growing together at very high supersaturations, Tomita et al. [22] have changed the frequency of the external $800 \mathrm{~V} \mathrm{~cm}^{-1} a c$-EF to range from 1 to $3 \mathrm{MHz}$. The reason for their choice is the difference between electrical permittivity of crystal polymorphs that depends on the intra-crystalline water contained in the crystals. (The possible effect of Ostwald's rule of stages has remained beyond the scope of the study.)

\subsection{EF Effects on Other Substances}

EFs also affect crystallization of other substances. Preferred crystal nucleation of glycine $\gamma$ polymorph has been observed by Aber et al. [48], who apply strong $d c$-EF to supersaturated aqueous glycine solution. The phenomenon is explained with the EF-induced orientation of the highly polar glycine molecules. Glycine polymorphism in the presence of a pulsed EF has also been explored by Di Profio et al. [49]. Their experimental results give grounds for the conclusion that the effect of different factors affecting glycine polymorphism can be graded as follows: $\mathrm{EF}>\mathrm{pH}>$ supersaturation level $>$ solute concentration. 
Hu et al. [50] and Parniakov et al. [51] have looked into the effects of pulsed EF energy on sucrose crystal nucleation in supersaturated solutions. Parniakov and coworkers report a significant reduction in crystal nucleation induction time when pulsed EFs are applied as compared to the untreated sample. For a high voltage electric discharge-assisted nucleation, the authors observe a decrease in the effective crystallization time and an increase in the maximum rate of crystallization when EF strength and pulse number is increased. They conclude that the dependence of nucleation rate on EF strength, number of pulses being applied, supersaturation of sucrose solutions and solution flow rate provides an opportunity to control the size distribution and structure of sucrose crystals.

To be functionally active, the proteins require a minimum amount of water. Therefore, it is essential to explore EF effect on the surrounding water. Since the protein itself has an internal dipole moment, an EF may evoke conformational changes to its molecule. The coupling effect of protein surface charge and protein hydration water on protein overall dipolar response plays an important role. The electric force exerted on the protein dipole results in a torque that will rotate the protein and is likely to affect the diffusion motions of the hydration water. To study the dynamics of lysozyme and its hydration water, Favi et al. [43] have used quasielastic neutron scattering measurements in the presence/absence of external static EFs $\left(\mathrm{D}_{2} \mathrm{O}\right.$ and $\mathrm{H}_{2} \mathrm{O}$ hydrated lysozyme is compared). The measurements reveal that the nano- to pico-second dynamics of the protein is unaffected by EF, possibly due to the stronger intra-molecular interactions compared to the maximum achieved field strength of 1 $\mathrm{kV} / \mathrm{mm}$. In addition, no appreciable quantitative enhancement of the diffusive dynamics of hydration water is observed. Finally, it is reported that EF of a solvated ion in water induces ordering in the surrounding water molecules, which, however, extends no further than several solvation shells [82].

As already mentioned, Koizumi et al. [27] have suggested that EF affects not only the protein molecules, but also the liquid entropy, probably because it impacts the water molecules. External EF makes them more polarized. Strong EF can also decrease hydrogen bond strength and even destruct such bonds in the water [83]. In other words, EF makes the water less structured. However, as noted above, the external EF is exponentially screened due to the high ionic strength of protein solutions that are under crystallization conditions. For the same reason, the electric double layer is relatively thin. Interestingly, static EF increases ice nucleation rate during crystallization of water. Theoretical calculations have shown that the application of such fields tends to decrease Gibbs free energy of the system, thus reducing the critical nucleus radius [84].

\section{Perspective}

Up to now, EF effects on growing protein crystals are studied by means of microscopic observations. The mesoscopic scale response of a crystallizing system to external EF can provide additional insights to the fine details of crystallization process under such conditions. A good candidate for this job seems to be the LCM-DIM, in combination with EF.

Enabling simultaneous in situ observation of single steps on a protein crystal surface and the crystal edges, LCM-DIM can render valuable information, distinguishing protein crystal growth from dissolution. Crystal dissolution starts from crystal edges, and the elementary surface steps propagate towards the center of the crystal face; and vice versa, growth is characterized by step propagation in reverse direction, towards the crystal edges. Therefore, growth and dissolution can be distinguished clearly by observing step propagation direction using LCM-DIM [54]. As is well-known, crystal surface growth occurs when supersaturation is imposed. With proteins having temperature dependent solubility, a crystal grows by setting solution temperature below (for normal solubility temperature dependence), respectively above (for retrograde solubility) the equilibrium temperature. Respectively, crystal dissolution occurs in the reverse cases, and the equilibrium temperature can be determined precisely; it corresponds to the case when the elementary surface steps do not move in either direction.

A quantitative rendition of the interrelation between EF effects and applied supersaturation is worth studying. Man obtains a decisive amount of information by seeing. Thus, an experimentum crucis would be imposing an EF on a preliminarily equilibrated (crystallizing) system, characterized 
by unmovable elementary surface steps. Switching on and off a $d c$-EF in a LCM-DIM observation will show whether EF has some impact on step dynamics or not. For instance, does EF energy [85] really change the equilibrium condition? The unambiguous answer to this question would be provided by a simple observation of the step behavior. If such steps start moving when applying a $d c-E F$, its impact will be obvious on a mesoscopic scale. If so, the sign and the amount of $\Delta \mu$ change that results from $\mathrm{EF}$ impact can be checked by observing whether the imposed EF prompts crystal growth or dissolution (respectively, whether EF accelerates or decelerates the movement of steps); and, if yes, to what extent? If such data are available, enthalpy and entropy of crystallization in presence of electric field can be calculated from the solubility, as described by Sleutel et al. [54]. It is of prime importance to check whether there is a change in this thermodynamic parameters that govern the crystallization process. (For fast crystal growth processes MI may be applied in a similar way.)

Likewise, EF effect (if any) on TSN mechanism of protein crystal nucleation and growth is worth exploring. It is to be hoped that the behavior of dynamic mesophases (amorphous metastable crystal precursors, according to TSN) under such conditions could be explained/predicted. In view of the importance of EF-impact, it is worth probing separately into protein crystal nucleation and growth. Detachment of the two consecutive stages, under simultaneous EF action, could be pursued. Extensive experimental observations as well as new ideas are needed to probe further into these issues.

Further research is required to clarify the effect that EF may exert on the protein crystallization by changing the hydration shells of protein molecules. At least a partial destruction of the letter is needed for crystal bond formation. It is the author's opinion, however, that the spatial distribution of water molecules in/around the protein lattice contacts depends on the kind of patches involved (hydrophilic or hydrophobic) and not so much on the EF. Computational approaches may help in elucidating the problem.

In addition, the complex response of different substances to EFs is worth studying to understand fully the mechanism of nucleation and crystal growth under such conditions [86].

\section{Conclusions}

Significant progress in studying the EF-assisted protein crystallization was achieved recently. The research in this field has been carried out using four main approaches, such as studies on the effects of external and internal EFs, by applying both $d c$ and $a c$. Despite the progress, due to the enormous complexity of the process under such conditions, a detailed and uniform comprehension of EF-assisted protein crystal nucleation is still lacking. In particular, numerous mesoscopic and molecular scale crystallization mechanism details remain unknown. Needless to say, further upgrading knowledge with new information from experimental studies using sophisticated methods is required to support broader and profounder insights into this issue. Such information can be acquired from more precise measurements using novel super-resolution techniques, e.g., the LCM-DIM and MI. In addition, an appropriate combination of known approaches may improve our insight in the EF-effect on the crystallization process.

Understanding crystal nucleation and growth under EFs is important as far as practical application is concerned, e.g., use of $d c$-voltage during crystallization of food systems [84] and for inducing crystallization and to control crystal forms [87]. The application of $d c$-voltage during preservation of food systems (especially freezing) offers a new perspective to the food industry; during the freezing process, the $d c$-voltage promotes ice nucleation at a higher temperature and reduces the induction time. The freezing process under static EF produces smaller ice crystals in the food products, resulting in less freeze damage; it is thus expected to minimize cell disruption, to lessen the protein denaturation, and finally to preserve the texture of the fresh food after thawing [84].

Acknowledgments: The author would like to acknowledge the contribution of COST Action CM1402 Crystallize. This work is co-financed by the National Science Fund of the Bulgarian Ministry of Education and Science, under contract DCOST 01/22. 
Conflicts of Interest: The author declares no conflict of interest. The founding sponsor had no role in the design of the study; in the collection, analyses, or interpretation of data; in the writing of the manuscript, and in the decision to publish the results.

\section{References and Notes}

1. Proteins enact a wide range of biological functions in the human body, such as catalysis, regulation, communication, mechanical support, movement, and transport.

2. For an excellent review on structure-guided drug discovery based on application of protein crystallography see "Blundell, T.L. Protein crystallography and drug discovery: Recollections of knowledge exchange between academia and industry. IUCrJ 2017, 4, 308-321.". The author would like to point out that his drive to explore topics such as crystalline insulin drug formulations, renin inhibitors, retroviral proteases (including AIDS antivirals), drug discovery in oncology and infectious disease, as well as for creating computer programs for generating reasonable protein structure models, has been significantly enhanced by the knowledge exchange between the pharmaceutical industry and academia.

3. Neutron crystallography requires growth of substantially larger protein crystals, greater than $0.1 \mathrm{~mm}^{3}$ in size are preferred, i.e., 4-5 orders of magnitude larger than those used in synchrotron X-ray data collection. The power of neutron crystallography consists in higher precision by visualization of $\mathrm{H}$-atoms (which play essential roles in macromolecular structure and catalysis), thus helping scientists to understand enzyme reaction mechanisms and hydrogen bonding.

4. Taleb, M.; Didierjean, C.; Jelsch, C.; Mangeot, J.P.; Capelle, B.; Aubry, A. Crystallization of proteins under an external electric field. J. Cryst. Growth 1999, 200, 575-582. [CrossRef]

5. Taleb, M.; Didierjean, C.; Jelsch, C.; Mangeot, J.P.; Aubry, A. Equilibrium kinetics of lysozyme crystallization under an external electric field. J. Cryst. Growth 2001, 232, 250-255. [CrossRef]

6. Mirkin, N.; Frontana-Uribe, B.A.; Rodriguez-Romero, A.; Hernandez-Santoyo, A.; Moreno, A. The influence of an internal electric field upon protein crystallization using the gel acupuncture method. Acta Crystallogr. 2003, D59, 1533-1538. [CrossRef]

7. Moreno, A.; Sazaki, G. The use of a new ad hoc growth cell with parallel electrodes for the nucleation control of lysozyme. J. Cryst. Growth 2004, 264, 438-444. [CrossRef]

8. Sazaki, G.; Moreno, A.; Nakajima, K. Novel coupling effects of the magnetic and electric fields on protein crystallization. J. Cryst. Growth 2004, 262, 499-502. [CrossRef]

9. Mirkin, N.; Jaconcic, J.; Stojanoff, V.; Moreno, A. High resolution X-ray crystallographic structure of bovine heart cytochrome $\mathrm{c}$ and its application to the design of an electron transfer biosensor. Proteins Struct. Funct. Bioinf. 2008, 70, 83-92. [CrossRef] [PubMed]

10. Frontana-Uribe, B.A.; Moreno, A. On electrochemically assisted protein crystallization and related methods. Cryst. Growth Des. 2008, 8, 4194-4199. [CrossRef]

11. Gil-Alvaradejo, G.; Ruiz-Arellano, R.R.; Owen, C.; Rodríguez-Romero, A.; Rudiño-Piñera, E.; Antwi, M.K.; Stojanoff, V.; Moreno, A. Novel protein crystal growth electrochemical cell for applications in X-ray diffraction and atomic force microscopy. Cryst. Growth Des. 2011, 11, 3917-3922. [CrossRef]

12. Espinoza-Montero, P.J.; Moreno-Narváez, M.E.; Frontana-Uribe, B.A.; Stojanoff, V.; Moreno, A. Investigations on the use of graphite electrodes using a hull-type growth cell for electrochemically assisted protein crystallization. Cryst. Growth Des. 2013, 13, 590-598. [CrossRef] [PubMed]

13. Flores-Hernandez, E.; Stojanoff, V.; Arreguin-Espinosa, R.; Moreno, A.; Sanchez-Puig, N. An electrically assisted device for protein crystallization in a vapor-diffusion setup. J. Appl. Crystallogr. 2013, 46, 832-834. [CrossRef] [PubMed]

14. Martínez-Caballero, S.; Cuéllar-Cruz, M.; Demitri, N.; Polentarutti, M.; Rodriguez-Romero, A.; Moreno, A. Glucose isomerase polymorphs obtained using an ad hoc protein crystallization temperature device and a growth cell applying an electric field. Cryst. Growth Des. 2016, 16, 1679-1686. [CrossRef]

15. Rodríguez-Romero, A.; Esturau-Escofet, N.; Pareja-Rivera, C.; Moreno, A. Crystal Growth of High-Quality Protein Crystals under the Presence of an Alternant Electric Field in Pulse-Wave Mode, and a Strong Magnetic Field with Radio Frequency Pulses Characterized by X-ray Diffraction. Crystals 2017, 7, 179. [CrossRef] 
16. Pareja-Rivera, C.; Cuéllar-Cruz, M.; Esturau-Escofet, N.; Demitri, N.; Polentarutti, M.; Stojanoff, V.; Moreno, A. Recent Advances in the Understanding of the Influence of Electric and Magnetic Fields on Protein Crystal Growth. Cryst. Growth Des. 2017, 17, 135-145. [CrossRef]

17. Moreno, A. Protein Crystallography, Methods and protocols. In Advanced Methods of Protein Crystallization; Wlodawer, A., Dauter, Z., Jaskolski, M., Eds.; Springer Protocols: Berlin, Germany, 2017; Chapter 3; pp. 51-76.

18. Koizumi, H.; Fujiwara, K.; Uda, S. Control of nucleation rate for tetragonal hen-egg white lysozyme crystals by application of an electric field with variable frequencies. Cryst. Growth Des. 2009, 9, 2420-2424. [CrossRef]

19. Koizumi, H.; Fujiwara, K.; Uda, S. Role of the electric double layer in controlling the nucleation rate for tetragonal hen egg white lysozyme crystals by application of an external electric field. Cryst. Growth Des. 2010, 1, 2591-2595. [CrossRef]

20. Koizumi, H.; Uda, S.; Fujiwara, K.; Nozawa, J. Effect of various precipitants on the nucleation rate of tetragonal hen egg-white lysozyme crystals in an AC external electric field. J. Cryst. Growth 2010, 312, 3503-3508. [CrossRef]

21. Koizumi, H.; Uda, S.; Fujiwara, K.; Nozawa, J. Control of effect on the nucleation rate for hen egg white lysozyme crystals under application of an external ac electric field. Langmuir 2011, 27, 8333-8338. [CrossRef] [PubMed]

22. Tomita, Y.; Koizumi, H.; Uda, S.; Fujiwara, K.; Nozawa, J. Control of Gibbs free energy relationship between hen egg white lysozyme polymorphs under application of an external alternating current electric field. J. Appl. Cryst. 2012, 45, 207-212. [CrossRef]

23. Koizumi, H.; Tomita, Y.; Uda, S.; Fujiwara, K.; Nozawa, J. Nucleation rate enhancement of porcine insulin by application of an external AC electric field. J. Cryst. Growth 2012, 352, 155-157. [CrossRef]

24. Koizumi, H.; Uda, S.; Fujiwara, K.; Tachibana, M.; Kojima, K.; Nozawa, J. Improvement of crystal quality for tetragonal hen egg white lysozyme crystals under application of an external alternating current electric field. J. Appl. Cryst. 2013, 46, 25-29. [CrossRef]

25. Koizumi, H.; Uda, S.; Fujiwara, K.; Tachibana, M.; Kojima, K.; Nozawa, J. Enhancement of crystal homogeneity of protein crystals under application of an external alternating current electric field. AIP Conf. Proc. 2014, 1618, 265-268.

26. Koizumi, H.; Uda, S.; Fujiwara, K.; Tachibana, M.; Kojima, K.; Nozawa, J. Control of subgrain formation in protein crystals by the application of an external electric field. Cryst. Growth Des. 2014, 14, 5662-5667. [CrossRef]

27. Koizumi, H.; Uda, S.; Fujiwara, K.; Tachibana, M.; Kojima, K.; Nozawa, J. Crystallization of high-quality protein crystals using an external electric field. J. Appl. Cryst. 2015, 48, 1507-1513. [CrossRef]

28. Koizumi, H.; Uda, S.; Fujiwara, K.; Tachibana, M.; Kojima, K.; Nozawa, J. Technique for high-quality protein crystal growth by control of subgrain formation under an external electric field. Crystals 2016, 6, 95. [CrossRef]

29. Koizumi, H.; Uda, S.; Fujiwara, K.; Okada, J.; Nozawa, J. Effect of an External Electric Field on the Kinetics of Dislocation-Free Growth of Tetragonal Hen Egg White Lysozyme Crystals. Crystals 2017, 7, 170. [CrossRef]

30. Hammadi, Z.; Astier, J.; Morin, R.; Veesler, S. Protein crystallization induced by a localized voltage. Cryst. Growth Des. 2007, 7, 1472-1475. [CrossRef]

31. Hammadi, Z.; Veesler, S. New approaches on crystallization under electric fields. Prog. Biophys. Mol. Biol. 2009, 101, 38-44. [CrossRef] [PubMed]

32. Hammadi, Z.; Astier, J.P.; Morin, R.; Veesler, S. Spatial and temporal control of nucleation by localized DC electric field. Cryst. Growth Des. 2009, 9, 3346-3347. [CrossRef]

33. Hammadi, Z.; Grossier, R.; Zhang, S.; Ikni, A.; Candoni, N.; Morin, R.; Veesler, S. Localizing and Inducing Primary Nucleation. Faraday Discuss. 2015, 179, 489-501. [CrossRef] [PubMed]

34. Al-Haq, M.; Lebrasseur, E.; Tsuchiya, H.; Torii, T. Protein crystallization under an electric field. Crystallogr. Rev. 2007, 13, 29-64. [CrossRef]

35. Wakamatsu, T.; Ohnishi, Y. Transparent cell for protein crystallization under low applied voltage. Jpn. J. Appl. Phys. 2011, 50, 48003. [CrossRef]

36. Wakamatsu, T.; Toyoshima, S.; Shimizu, H. Observation of electric-field induced aggregation in crystallizing protein solutions by forward light scattering. Appl. Phys. Lett. 2011, 99, 153701. [CrossRef]

37. Wakamatsu, T. Method and apparatus for characterization of electric field-induced aggregation in pre-crystalline protein solutions. Rev. Sci. Instrum. 2015, 86, 15112. [CrossRef] [PubMed] 
38. Wakamatsu, T. Low Applied Voltage Effects on Thaumatin Protein Crystallization. Trans. Mater. Res. Soc. Jpn. 2016, 41, 13-15. [CrossRef]

39. Nanev, C.N.; Penkova, A. Nucleation of lysozyme crystals under external electric and ultrasonic fields. J. Cryst. Growth 2001, 232, 285-293. [CrossRef]

40. Penkova, A.; Gliko, O.; Dimitrov, I.; Hodjaoglu, F.; Nanev, C.; Vekilov, P. Enhancement and suppression of protein crystal nucleation due to electrically driven convection. J. Cryst. Growth 2005, 275, 1527-1532. [CrossRef]

41. Rubin, E.; Owen, C.; Stojanoff, V. Crystallization under an external electric field: A case study of glucose isomerase. Crystals 2017, 7, 206. [CrossRef]

42. Li, F.; Lakerveld, R. The influence of alternating electric fields on protein crystallization in microfluidic devices with patterned electrodes in a parallel-plate configuration. Cryst. Growth Des. 2017, 17, 3062-3070. [CrossRef]

43. Favi, P.M.; Zhang, Q.; O'Neill, H.; Mamontov, E.; Diallo, S.O. Dynamics of lysozyme and its hydration water under an electric field. J. Biol. Phys. 2014, 40, 167-178. [CrossRef] [PubMed]

44. Kwokal, A.; Roberts, K.J. Direction of the polymorphic form of entacapone using an electrochemical tuneable surface template. CrystEngComm 2014, 16, 3487-3493. [CrossRef]

45. Pan, W.; Xu, H.; Zhang, R.; Xu, J.; Tsukamoto, K.; Han, J.; Li, A. The influence of low frequency of external electric field on nucleation enhancement of hen egg-white lysozyme (HEWL). J. Cryst. Growth 2015, 428, 35-39. [CrossRef]

46. Al-Haq, M.; Lebrasseur, E.; Choi, W.; Tsuchiya, H.; Torii, T.; Yamazaki, H.; Shinohara, E. An apparatus for electric-field-induced protein crystallization. J. Appl. Crystallogr. 2007, 40, 199-201. [CrossRef]

47. Hou, D.; Chang, H.C. Ac field enhanced protein crystallization. Appl. Phys. Lett. 2008, 92, 223902. [CrossRef]

48. Aber, J.E.; Arnold, S.; Ward, M.D.; Garetz, B.A.; Myerson, A.S. Strong dc electric field applied to supersaturated aqueous glycine solution induces nucleation of the $\gamma$ polymorph. Phys. Rev. Lett. 2005, 94, 145503. [CrossRef] [PubMed]

49. Di Profio, G.; Reijonen, M.T.; Caliandro, R.; Guagliardi, A.; Curcio, E.; Drioli, E. Insights into the polymorphism of glycine: Membrane crystallization in an electric field. Phys. Chem. Chem. Phys. 2013, 15, 9271-9280. [CrossRef] [PubMed]

50. Hu, B.; Huang, K.; Zhang, P.; Zhong, X.Z. Pulsed electric field effects on sucrose nucleation at low supersaturation. Sugar Tech 2015, 17, 77-84. [CrossRef]

51. Parniakov, O.; Adda, P.; Bals, O.; Lebovka, N.; Vorobiev, E. Effects of pulsed electric energy on sucrose nucleation in supersaturated solutions. J. Food Eng. 2017, 199, 19-26. [CrossRef]

52. Giege, R. What macromolecular crystallogenesis tells us-What is needed in the future. IUCrJ 2017, 4, 340-349. [CrossRef] [PubMed]

53. These techniques are applied because the resolution of the light microscope is limited to 200-300 nm (Abbe theory). Therefore, mesoscopic and molecular scale protein crystallization peculiarities are invisible by the conventional microscopy.

54. Sleutel, M.; Maes, D.; Van Driessche, A. Kinetics and Thermodynamics of Multistep Nucleation and Self-Assembly in Nanoscale Materials. In Advances in Chemical Physics; Nicolis, G., Maes, D., Eds.; Wiley-Blackwell: Malden, MA, USA, 2012; Volume 151, pp. 223-276.

55. It should be kept in mind, however, that supersaturations used to produce protein crystals suitable for X-ray diffraction are significantly higher than supersaturations that are optimal for AFM and LCM-DIM.

56. Yamazaki, T.; Kimura, Y.; Vekilov, P.G.; Furukawa, E.; Shirai, M.; Matsumoto, H.; Van Driessche, A.E.S.; Tsukamoto, K. Two types of amorphous protein particles facilitate crystal nucleation. Proc. Natl. Acad. Sci. USA 2017, 114, 2154-2159. [CrossRef] [PubMed]

57. Nanev, C.N. On some aspects of crystallization process energetics, logistic new phase nucleation kinetics, crystal size distribution and Ostwald ripening. J. Appl. Cryst. 2017, 50, 1021-1027. [CrossRef]

58. Nanev, C.N. Phenomenological consideration of protein crystal nucleation; the physics and biochemistry behind the phenomenon. Crystals 2017, 7, 193. [CrossRef]

59. Malkin, A.J.; Kuznetsov, Y.G.; McPherson, A. In Situ atomic force microscopy studies of surface morphology, growth kinetics, defect structure and dissolution in macromolecular crystallization. J. Cryst. Growth 1999, 196, 471-488. [CrossRef] 
60. McPherson, A.; Kuznetsov, Y.G. Mechanisms, kinetics, impurities and defects: Consequences in macromolecular crystallization. Acta Crystallogr. Sect. F Struct. Biol. 2014, 70, 384-403. [CrossRef] [PubMed]

61. Van Driessche, A.E.S.; Sazaki, G.; Otálora, F.; Gonza'lez-Rico, F.M.; Dold, P.; Tsukamoto, K.; Nakajima, K. Direct and noninvasive observation of two-dimensional nucleation behavior of protein crystals by advanced optical microscopy. Cryst. Growth Des. 2007, 7, 1980-1987. [CrossRef]

62. Suzuki, Y.; Sazaki, G.; Matsumoto, M.; Nagasawa, M.; Nakajima, K.; Tamur, K. First direct observation of elementary steps on the surfaces of glucose isomerase crystals under high pressure. Cryst. Growth Des. 2009, 9, 4289-4295. [CrossRef]

63. Gibbs, J.W. On the Equilibrium of Heterogeneous Substances. Trans. Connect. Acad. 1879, 3, 108-248, 343-524. [CrossRef]

64. Ten Wolde, P.R.; Frenkel, D. Enhancement of protein crystal nucleation by critical density fluctuations. Science 1997, 277, 1975-1978. [CrossRef] [PubMed]

65. Gebauer, D.; Kellermeier, M.; Gale, J.D.; Bergström, L.; Cölfen, H. Pre-nucleation clusters as solute precursors in crystallization. Chem. Soc. Rev. 2014, 43, 2348-2371. [CrossRef] [PubMed]

66. Whitelam, S. Control of Pathways and Yields of Protein Crystallization through the Interplay of Nonspecific and Specific Attractions. Phys. Rev. Lett. 2010, 105, 88102. [CrossRef] [PubMed]

67. Ferreira, C.; Barbosa, S.; Taboada, P.; Rocha, F.A.; Damas, A.M.; Martins, P.M. The nucleation of protein crystals as a race against time with on-and off-pathways. J. Appl. Cryst. 2017, 50, 1056-1065. [CrossRef]

68. Vekilov, P.G. Nucleation of protein crystals. Prog. Cryst. Growth Charact. Mater. 2016, 62, 136-154. [CrossRef]

69. Vivares, D.; Kaler, E.; Lenhoff, A. Quantitative imaging by confocal scanning fluorescence microscopy of protein crystallization via liquid-liquid phase separation. Acta Crystallogr. D Biol. Crystallogr. 2005, 61, 819-825. [CrossRef] [PubMed]

70. Sauter, A.; Roosen-Runge, F.; Zhang, F.; Lotze, G.; Jacobs, R.M.J.; Schreiber, F. Real-time observation of nonclassical protein crystallization kinetics. J. Am. Chem. Soc. 2015, 137, 1485-1491. [CrossRef] [PubMed]

71. Schubert, R.; Meyer, A.; Baitan, D.; Dierks, K.; Perbandt, M.; Betzel, C. Real-time observation of protein dense liquid cluster evolution during nucleation in protein crystallization. Cryst. Growth Des. 2017, 17, 954-958. [CrossRef]

72. Sleutel, M.; Van Driessche, A.E.S. Role of clusters in nonclassical nucleation and growth of protein crystals. Proc. Natl. Acad. Sci. USA 2014, 111, E546-E553. [CrossRef] [PubMed]

73. Sleutel, M.; Lutsko, J.; Van Driessche, A.E.S.; Duran-Olivencia, M.A.; Maes, D. Observing classical nucleation theory at work by monitoring phase transitions with molecular precision. Nat. Commun. 2014, 5, 5598. [CrossRef] [PubMed]

74. Nanev, C.N. Kinetics and Intimate Mechanism of Protein Crystal Nucleation. Prog. Cryst. Growth Charact. Mater. 2013, 59, 133-169. [CrossRef]

75. This withstanding, cryo-TEM images also have shown that there is a second type of non-crystalline particles assembling lysozyme, which appear on amorphous solid particle or a container wall (thus, highlighting the role of heterogeneous nucleation), and that lysozyme crystals appear within such particles.

76. Kellermeier, M.; Raiteri, P.; Berg, J.; Kempter, A.; Gale, J.; Gebauer, D. Entropy drives calcium carbonate ion association. Chemphyschem 2016, 17, 3535-3541. [CrossRef] [PubMed]

77. Jiang, Y.; Kellermeier, M.; Gebaue, D.; Lu, Z.; Rosenberg, R.; Moise, A.; Przybylski, M.; Cölfen, H. Growth of organic crystals via attachment and transformation of nanoscopic precursors. Nat. Commun. 2017, 8, 15933. [CrossRef] [PubMed]

78. Sleutel, M.; Vanhee, C.; Van de Weerdt, C.; Decanniere, K.; Maes, D.; Wyns, L.; Willaert, R. The Role of Surface Diffusion in the Growth Mechanism of Triosephosphate Isomerase Crystals. Cryst. Growth Des. 2008, 8, 1173-1180. [CrossRef]

79. Sleutel, M.; Maes, D.; Wyns, L.; Willaert, R. Kinetic roughening of glucose isomerase crystals. Cryst. Growth Des. 2008, 8, 4409-4414. [CrossRef]

80. Anderson, M.W.; Gebbie-Rayet, J.T.; Hill, A.R.; Farida, N.; Attfield, M.P.; Cubillas, P.; Blatov, V.A.; Proserpio, D.M.; Akporiaye, D.; Arstad, B.; et al. Predicting crystal growth via a unified kinetic three-dimensional partition model. Nature 2017, 544, 456-459. [CrossRef] [PubMed]

81. For an extremely lucid and contemporary explanation of what entropy is see "Nishinaga, T. Thermodynamics -for understanding crystal growth-. Prog. Cryst. Growth Charact. Mater. 2016, 62, 43-57.". 
82. Wilkins, D.M.; Manolopoulos, D.E.; Roke, S.; Ceriotti, M. Mean-field theory of water-water correlations in electrolyte solutions. J. Chem. Phys. 2017, 146, 181103. [CrossRef]

83. Shevkunov, S.V.; Vegiri, A. Electric field induced transitions in water clusters. J. Mol. Struct. THEOCHEM 2002, 593, 19-32. [CrossRef]

84. Jha, P.K.; Sadot, M.; Vinoa, S.A.; Jury, V.; Curet-Ploquina, S.; Rouaud, O.; Havet, M.; Le-Bail, A. A review on effect of DC voltage on crystallization process in food systems. Innov. Food Sci. Emerg. 2017, 42, 204-219. [CrossRef]

85. The potential energy of a continuous charge distribution is stored in the EF.

86. Han, B.; Chen, Z.; Louhi-Kultanen, M. Effect of a pulsed electric field on the synthesis of $\mathrm{TiO}_{2}$ and its photocatalytic performance under visible light irradiation. Powder Technol. 2017, 307, 137-144. [CrossRef]

87. Garetz, B.A.; Myerson, A.S.; Arnold, S.; Aber, J.E. Method for Using a Static Electric Field to Induce Crystallization and to Control Crystal Form. U.S. Patent 7,879,115 B2, 13 April 2004.

C 2017 by the author. Licensee MDPI, Basel, Switzerland. This article is an open access article distributed under the terms and conditions of the Creative Commons Attribution (CC BY) license (http:/ / creativecommons.org/licenses/by/4.0/). 